\title{
Three-dimensional spectroscopy with a fiber-fed NUV spectrograph
}

Robert Grange, Bruno Milliard, Ryan McLean, Christopher Martin, Jean Michel Deharveng, et al.

Robert Grange, Bruno Milliard, Ryan McLean, Christopher Martin, Jean Michel Deharveng, David Schiminovich, "Three-dimensional spectroscopy with a fiber-fed NUV spectrograph," Proc. SPIE 5898, UV, X-Ray, and Gamma-Ray Space Instrumentation for Astronomy XIV, 589812 (18 August 2005); doi: 10.1117/12.616934

SPIE. Event: Optics and Photonics 2005, 2005, San Diego, California, United States 


\title{
Three-dimensional spectroscopy with a fiber-fed NUV spectrograph
}

\author{
Robert Grange ${ }^{\mathrm{a}}$, Bruno Milliard ${ }^{\mathrm{a}}$, Ryan McLean ${ }^{\mathrm{b}}$, Christopher Martin $^{\mathrm{b}}$, Jean Michel Deharveng ${ }^{\mathrm{a}}$, \\ David Schiminovich ${ }^{\mathrm{C}}$, \\ a'Laboratoire d'Astrophysique de Marseille, Allée Peiresc, 13012 Marseille France ; \\ ${ }^{\mathrm{b} C a l i f o r n i a}$ Institute of Technology, Pasadena, CA, 91125 USA; \\ ${ }^{\circ}$ Columbia University, New York, NY 10027 USA
}

\begin{abstract}
In the context of the NASA balloon borne experiment named Fireball (Faint Intergalactic Redshifted Emission BALLoon) dedicated to map the Intergalactic Medium, we designed a fiber-fed near ultraviolet spectrograph to work in the $200 \mathrm{~nm}$ atmospheric transmission window. We first describe the system level optimization leading to the atypical use in UV of a fiber Integral Field Unit at the focus of a one meter diameter parabolic mirror. For the qualification of the design we measured the absolute transmission of an UV polyimide 100 microns core fiber. The fiber bundle made of 400 fibers rearranged in a $50 \mathrm{~mm}$ slit feeds an F/2.5 spectrograph based on an Offner Littrow mount. We present the optical performances of this design with a high throughput and a well matched aperture ratio.
\end{abstract}

Keywords: UV spectrograph, optical fiber, integral field unit

\section{INTRODUCTION}

The NASA ballon borne experiment named Fireball (Faint Intergalactic Redshifted Emission BALLoon) to be launched in summer 2007, is designed to map the Warm Hot Intergalactic Medium (WHIM) in the near ultraviolet region $(200 \mathrm{~nm})$ allowed by the atmospheric window at the typical $40 \mathrm{~km}$ flight balloon altitude. The WHIM is predicted to contain a significant fraction of the baryons at low and moderate redshift and should be detected by the redshifted OVI line (1032 A), emitted by filamentary structure of typical angular width of 8 arc second. The requirement of a very high sensitivity to extended sources coupled to a 8 arc second angular resolution leads to an optical system with one meter diameter pupil and 2.5 meter focal length. The typical 2-3 arc min extension of the WHIM structure yields to adopt an Integral Field Unit (IFU) that is much more efficient than a long slit used in push broom mode. The spectrograph resolution power of 5000 is linked to the requirement of not decreasing the contrast of the narrow emission line over the continuum UV background.

In this paper we will describe the system level optimization, the absolute transmission measurement of a fiber in the near ultraviolet and finally the design of the compact high sensitivity spectrograph.

\section{SYSTEM LEVEL OPTIMIZATION}

In the process of the Fireball system level optimization we try to find the simplest solution with the minimum number of optical elements to guarantee a high throughput in the $200 \mathrm{~nm}$ window.

Among different possible telescope and spectrograph designs we finally adopted a simple one where an F/2.5 one meter diameter parabolic mirror feeds a fiber IFU. Several ground based experiments in the visible are based on fiber $\mathrm{IFU}^{1,2}$ without lens array. For Fireball the $160 \operatorname{arcsec}^{\wedge} 2$ field of view is rearranged into a $50 \mathrm{~mm}$ slit height by a coherent 400 fiber bundle. A fiber core diameter of 100 microns ( 8 arc second) combined with a 110 microns diameter cladding and a 125 microns diameter polyimide buffer allows a reasonable packaging density at the slit level. The packaging density at the bundle entrance level (field of view) which is the standard IFU criterion is not the critical point here since Fireball is designed to map disseminated and not compact structures. In fact the limitation is at the detector level where

UV, X-Ray, and Gamma-Ray Space Instrumentation for Astronomy XIV edited by Oswald H. W. Siegmund, Proc. of SPIE Vol. 5898 589812, (2005) · 0277-786X/05/\$15 · doi: 10.1117/12.616934

Proc. of SPIE Vol. 5898 589812-1 
each fiber will be separated by 25 microns.

High $\mathrm{OH}$ silica multimode fiber with enhanced properties in deep UV appears to be the best choice for the Fireball application. At first glance the choice of an IFU based on fibers instead of slicer mirrors in the near ultraviolet seems unexpected but the fewer optics involved in the fiber design largely compensate the inherent low efficiency transmission of a fiber in the ultraviolet. The fiber core diameter well matches the spatial resolution avoiding the use of magnifying and demagnifying optical train. In our case the standard numerical aperture of fibers $(\mathrm{NA}=0.22$ corresponding to $F / 2.3$ ) well matches the numerical aperture of the primary mirror and spectrograph. As a consequence no lens array is needed in the entrance nor in the output of the fiber bundle and then the system is simple to build and very efficient in the $195-210 \mathrm{~nm}$ ultraviolet region of interest.

We first thought of a direct pointing system where the telescope and its associated spectrograph were attached to an alt-azimuthal gimbal. But it appeared rapidly that this direct pointing system led to either a solution with too high inertia or too long fiber bundle. The reduction of the pointed instrument inertia by locating the spectrograph close to the gimbal axis but far from the parabolic focus implies to have a typical 3 meters long fiber with significant throughput degradation. Adding a secondary mirror would imply to increase the speed of the primary by a factor of 2 or 3 leading to a quasi unfeasible primary within the budget of a balloon-borne mission. On the other hand a short fiber length would imply a $60 \mathrm{~kg}$ spectrograph 2 meters away from the gimbal axis with a prohibitive inertia. We finally adopted a siderostat mount where a $1.2 \mathrm{~m}$ diameter flat mirror reflect the sky to a fixed 1 meter parabolic mirror attached to the top of the gondola. The flat siderostat has a $150 \mathrm{~mm}$ diameter central hole and the parabola focus is located $150 \mathrm{~mm}$ behind the rear face of the siderostat. This design shown in figure 1 can accommodate an efficient half meter fiber length with a very low inertia siderostat.

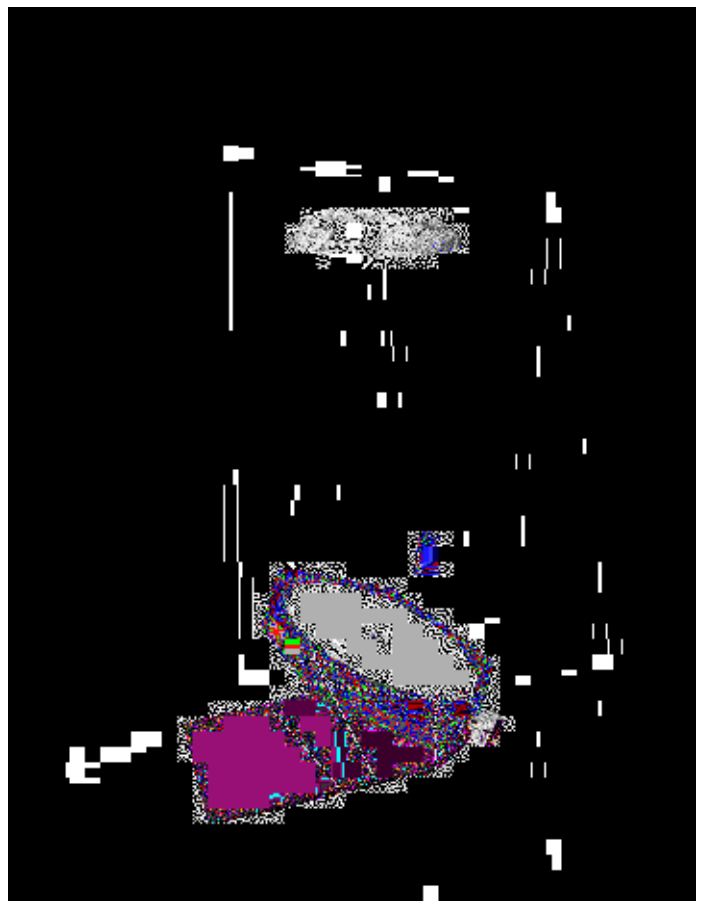

Figure 1: Schematic of the proposed gondola

\section{MEASUREMENT OF THE FIBER ABSOLUTE TRANSMISSION IN THE NUV}

The $200 \mathrm{~nm}$ region is unfortunately very close to the cutoff of a silica high $\mathrm{OH}$ multimode fiber. The transmission therefore degrades rapidly below $250 \mathrm{~nm}$ mainly due to the Rayleigh scattering. Since the fiber efficiency is a key parameter for our design and absolute measurements of fiber transmission at $200 \mathrm{~nm}$ are not currently available, we 
developed our own measurement bench in order to qualify our design.

One of the difficulties of UV measurement is the visible straylight level compared to the weak flux of lines available around $200 \mathrm{~nm}$. We had in house a $400 \mathrm{~mm}$ diameter UV telescope at F/2.5 designed for a previous balloon borne experiment. In addition to its well matched aperture ratio this telescope has two multilayer UV coated mirrors which combine with the CsTe photocathode of the imaging photon counting to produce a strong rejection of the visible light.

The principle of the absolute measurement is to compare the flux going out a 100 microns pinhole to the flux going out a 100 microns core fiber assuming the same uniform F/2.5 injection beam. The diameter of the injection beam is larger than 100 microns to minimize centering problem. Nevertheless for each measurement we find the maximum flux by precisely adjusting the orientation of the telescope holding the fiber. The optical bench is equipped with a hydrogen discharge lamp and a monochromator with a 2000 microns diameter exit hole. A 8 meter focal length collimator produce a $400 \mathrm{~mm}$ parallel beam to feed the UV F/2.5 telescope which produces the right injection beam with a diameter of 250 microns. The optical setup is located in a vacuum chamber but we did measurements at the atmospheric pressure knowing that wavelengths down to $195 \mathrm{~nm}$ could be reached under this condition.

We used a $25 \mathrm{~mm}$ CsTe photon counting detector to measure the flux at the exit of the pinhole or the fiber with no relay optics. To deduce the absorption in $\mathrm{dB} / \mathrm{m}$ we tested several length of the same unstripped fiber by successive cleaving of the exit face leaving the cleaved entrance face unchanged. It appears during the tests that stripping the polyimide is a difficult task which often results in damaging the fiber thus reducing the transmission. The fast cleaving process was well adapted for the measurement of one fiber but for the Fireball flight bundle we envision unstripped polyimide fibers bonded in custom ferrules with both ends polished.

Deduced from our measurements at four different lengths of the same Ceramoptec fiber at $202.5 \mathrm{~nm}$, the absolute transmission can be calculated as function of the fiber length by:

$$
\text { Absolute Transmission }=10^{\wedge}\left(-0,2115^{*} \mathrm{~L}-0,0173\right) \quad \text { where } \mathrm{L} \text { is the fiber length. }
$$

As a result a half meter 100/110/125 microns UV polyimide fiber could offer $75 \%$ absolute transmission including the Fresnel loss at both ends.

\section{SPECTROGRAPH OPTICAL DESIGN}

The optical requirements of the Fireball spectrograph are quite challenging: a resolution power of 5000 , an $\mathrm{F} / 2.5$ aperture ratio, a slit height of $50 \mathrm{~mm}$, all this through a minimum number of reflective optics. It rapidly turns out that an all reflective Offner mount with two concentric spherical mirrors could be a good baseline with its inherent correction of the spherical aberration, coma, astigmatism and field curvature. Mertz ${ }^{3}$ and $\mathrm{Lobb}^{4}$ studied this type of fast concentric spectrograph where a convex reflective grating replaces the secondary mirror of a concentric Offner relay with unit magnification. For the high resolution power of Fireball we adopted this mount used in Littrow mode where the angle of incidence on the grating is equal to the angle of diffraction. As a consequence the diffracted beam reflects on the same portion of the primary mirror and comes back close to the entrance slit. The F/2.5 focal ratio imposes to work with a highly decentered field and thus higher order astigmatism appears; this can be reduced following Ih and Yen ${ }^{5}$, by a slight decenter of the grating and by changing its distance to the spherical primary. The spectrograph optical design is shown in figure 2. 


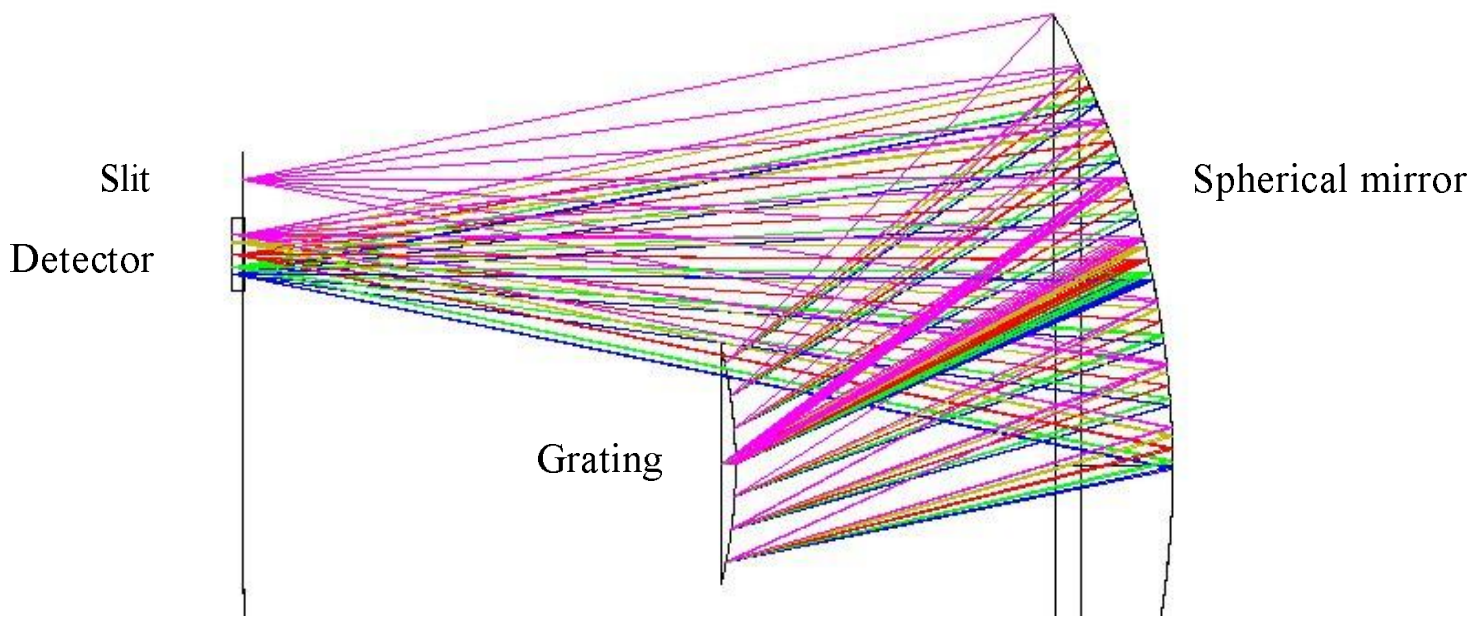

Figure 2: Layout of the Offner Spectrograph with a convex holographic grating used in Littrow mode

The convex holographic spherical grating with parallel straight grooves has a groove density of $5000 \mathrm{~g} / \mathrm{mm}$ and its feasibility has been checked with the Jobin-Yvon company (Paris). With its high groove density and angular conditions the grating will work in the electromagnetic regime with diffracted order 1 and zero only. This grating regime will insure a potential high efficiency even with unblazed sinusoidal groove shape. The remaining photoresist layer on the grating will be coated with $\mathrm{Al} \mathrm{MgF} 2$. Figure 3 shows the spot diagrams of the fireball spectrograph for five wavelengths in the bandpass.

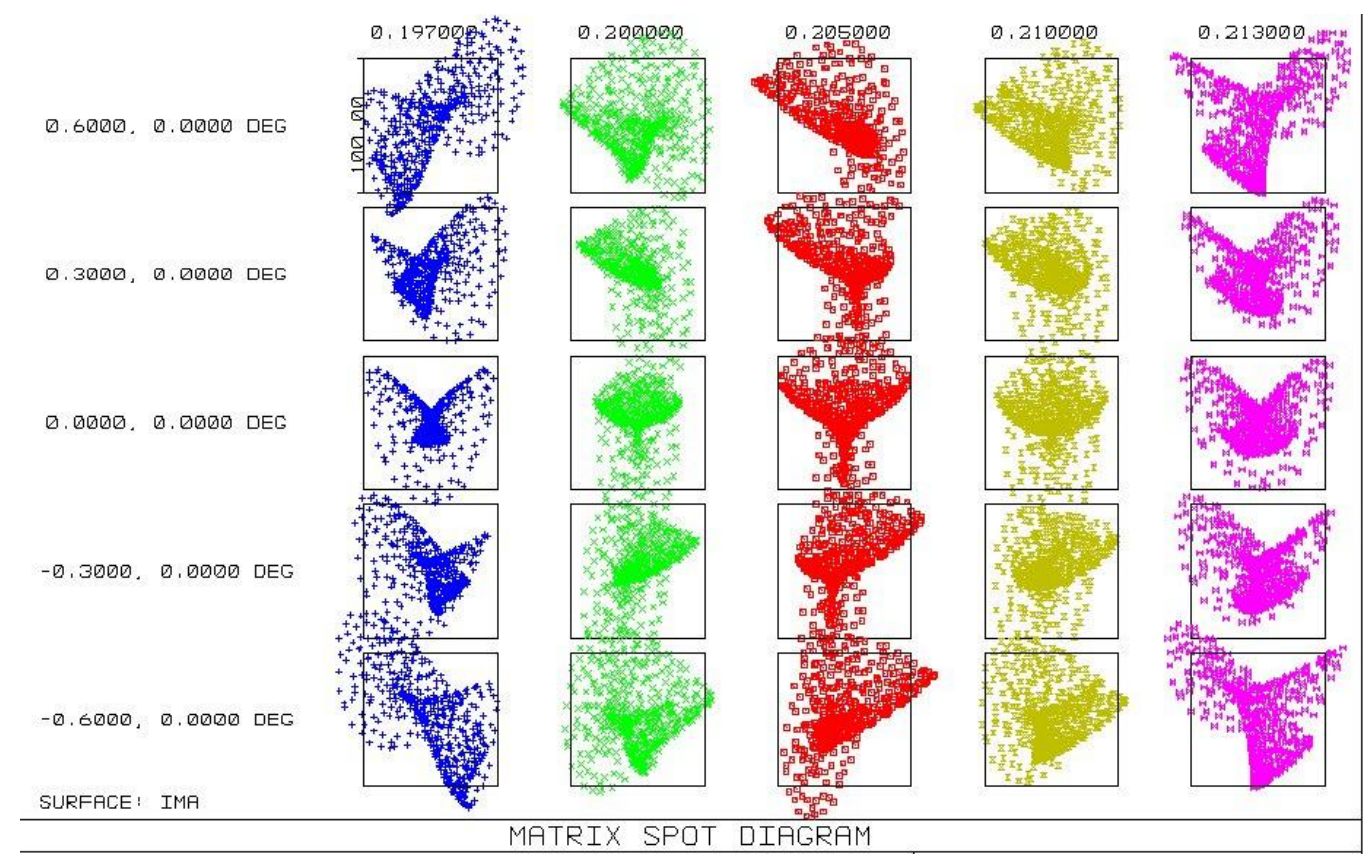

Figure 3: Spot Diagram for different slit heights in the full bandpass (side of the square $=100$ microns) 


\section{CONCLUSION}

The Fireball scientific goal of mapping the WHIM in the $200 \mathrm{~nm}$ region requires an especially challenging sensitivity combined with 8 arc second spatial resolution and a spectral resolution of 5000 . The system level optimization of Fireball (maximizing UV throughput and simplicity of the pointing system) leads us to implement a fiber IFU feeding a fast spectrograph. Compared to an IFU based on slicer mirrors, a fiber IFU reduces drastically the number of optical elements since the fiber core diameter well matches the spatial resolution avoiding the use of additional optics. We will also use no optics at both ends of the fiber bundle since the F/2.5 telescope and the spectrograph have the same aperture ratio close to the standard numerical aperture of fiber (NA: 0.22 ). We measured at $202.5 \mathrm{~nm}$ a half meter fiber that has an absolute transmission of $75 \%$. The spectral resolution of 5000 is reached by designing a concentric spectrograph based on an Offner mount with a $5000 \mathrm{~g} / \mathrm{mm}$ holographic grating used in Littrow mode.

\section{REFERENCES}

1. Arribas S, Mediavilla E and Rasilla J L 1991 Astrophys. J. 369260

2. Barden S and Wade R 1988 ASP Conf. Ser. 3113

3. L. Mertz, "Concentric spectrographs," Appl. Opt. 16, 3122-3124 (1977)

4. D. R. Lobb, “Theory of concentric designs for grating spectrometers," Appl. Opt. 33, 2648-2658 (1994)

5. C. S. Th and K. Yen, "Imaging and Fourier transform properties of an all-spherical-mirror system," Appl. Opt. 19, 4196-4199 (1980) 